\title{
Role of KOICA Project in Development of Nuclear Medicine in Bangladesh
}

\author{
Prof. Dr. Shamim Momtaz Ferdousi Begum
}

National Institute of Nuclear Medicine and Allied Sciences (NINMAS)

Correspondence Address: Prof. Dr Shamim Momtaz Ferdousi Begum, Professor and Head of PET-CT Division, National Institute of Nuclear Medicine and Allied Sciences (NINMAS) BAEC, Dhaka, Bangladesh, Email: shamimmomtaz23@gmail.com

Bangladesh has a long history of governance structure of nuclear medicine (NM) practice comprising one National Institute of Nuclear Medicine and Allied Sciences (NINMAS, previously it was INM) and fourteen Institutes of Nuclear Medicine \& Allied Sciences (INMAS). Around one hundred nuclear medicine physicians, few physicists and quite significant number of technologists are working in these institutes. Almost all of the NM institutes of the country are equipped with the facilities of one or two Gamma cameras, SPECT; SPECT-CT machines, has basic infrastructure and trained NM personnel, who are providing good health services to the country.

During the last decade NM practice has remarkably developed in the country with significant advancement in instrumentation, radio-pharmacy and information technology. Currently some institutes of NMof the country are equipped with state-of-art nuclear medicine instruments. Most of the NM personnel were well trained in the past environment of NM, when the new instruments of PET-CT or SPECT- CT were unavailable. Lack of skilled NM personnel is a major challenge for survival and proper utilization of the new technology. Quality training programs for NM personnel are indispensible to develop skill and competency. Knowledge and performancesof them should be enhanced by training in an appropriate learning environment of the state-of-the-art. Thereby they can adapt themselves to do the state-of-art therapy and imaging. They will be able to get connected with the rapidly-evolving disciplines of their own, nuclear medicine.

Keeping these issues in mind, a good friend of Bangladesh NM, Prof. Dong Soo Lee, Professor of Department of Nuclear Medicine, Department of Molecular Medicine and Biopharmaceutical Science,
Seoul National University (SNU), President of World Federation of Nuclear Medicine and Biology (WFNMB) has taken two international projects funded by Seoul National University, Korean International Cooperation Agency Project (SNU, KOICA) and Asi@Connect project.

The SNU's KOICA project is titled "Infrastructure Strengthening Project of Nuclear Medicine in Bangladesh for Treatment of Non-communicable Disease". This is a three years duration project (from 2018 to 2020), compliance with SDGs goals 3.4, 3.c. The implementing organizations are Korea: Seoul National University R\&DB Foundation and partner country is Bangladesh through NINMAS. Development of human infrastructure, remote seminar or education system in NM field is the overall objective of this project. The Asi@Connect project is titled "Enhancement of Nuclear Medicine Performance using Asi@Connect in Asian Countries". This is a two years duration (2018-2019) project and involved countries are Korea, Bangladesh, Indonesia and Vietnam. The overall objective of this project is to build up national and international network connections and communication to enhance academic, clinical and research excellence in partner counties.

To achieve the project objectives several activities are included in the work plan; like long term (one year duration ) training for the rising NM physicians and physicists or radio-chemists at Korea, on-site workshop at Korea and Bangladesh for re-education of the opinion leaders of each country's NM society. Build up of high speed network connection to medical users of each country to get connected with TEIN and enable NM experts with international colleagues using the networks. 
After one year of the project,several achievements are evident, like three rising NM physicians and one physicist are obtaining their training from Seoul National University, Chonnam National University and Kyungpook National University, Korea. Another two rising NM physicians, one physicist and one radio chemist have received their nomination of long term training for the next phase. Nuclear medicine leaders were invited at a workshop held in Korea on July 2018 on "Multidisciplinary Team Approach in Cancer Management". Five senior NM physicians participated from Bangladesh. Inspired from that workshop, NINMAS has developed a Multidisciplinary Expert Team (MDT) for management of complicated cases of thyroid cancer including oncologists, radiation oncologists,

otolaryngology \& head-neck surgeons, endocrinologists, palliative care medicine specialists, pathologists, radiologists, endocrinologists and leading nuclear medicine physicians. The first MDT meeting was held in November 2018 and subsequent monthly meeting are being held. A national workshop "KOICA Education Workshop and Remote Seminar of Nuclear Science" was held at NINMAS Dhaka from 9th to 12th December, 2018 with visiting speakers from SNU including Prof. Dong Soo Lee. Twenty seven participants participated in the workshop from different backgrounds like NM physicians, physicists and radio chemists from all over Bangladesh.

Another achievement is installation of video conference system between NINMAS and other INMASes. NINMAS is now connected with two INMASes within Dhaka city and INMASes of remote cities of the country like Mymensingh, Rajshahi, and Khulna. The deliverable hard ware (router, video conferencing system; camera, speaker) and software (Vidyo) were provided by the project to the NM institutes.

The 5th Theranostics World Congress 2019 will be held in Jeju Island, South Korea on 1-3 March. Seven abstracts are accepted and will be presented in conference by Bangladesh fellows. One of our leading Professors is invited as a speaker in panel discussion and she is also honored as a member of Scientific Committee of the congress. This meeting will provide the opportunity to the Bangladeshi young generation to gather together and update the future direction of nuclear medicine and nuclear theranostics.

It is expected that after completion of the project there will be direct beneficiaries of 87 nuclear medicine personnel, 1690 indirect beneficiaries and a significant number of representative beneficiaries through presentations at international conference. 